\title{
El género Pluchea (familia Asteraceae, tribu Plucheeae) en México
}

\section{The genus Pluchea (family Asteraceae, tribe Plucheeae) in Mexico}

\author{
José Luis Villaseñor ${ }^{1 *}$ y José Ángel Villarreal² \\ ${ }^{1}$ Departamento de Botánica, Instituto de Biología, UNAM, Apartado postal 70-367, 04510 México, D. F. \\ *Correspondencia: vrios@ibiologia.unam.mx \\ ${ }^{2}$ Universidad Autónoma Agraria Antonio Narro, Departamento de Botánica. 25315 Buenavista, Saltillo, Coahuila. javillarreal00@hotmail.com
}

Resumen. El género Pluchea (Asteraceae, Plucheeae), distribuido principalmente en las regiones tropicales de América, África, Asia y Australia, registra en México 9 especies, 2 de ellas endémicas del país. Se presenta una sinopsis del género para México, incluyendo una clave para la identificación de las especies y mapas de distribución obtenidos de los ejemplares revisados.

Palabras clave: Asteraceae, Pluchea, Plucheeae, México.

\begin{abstract}
The genus Pluchea (Asteraceae, Plucheeae) is mostly found in the tropical regions of America, Africa, Asia, and Australia; in Mexico the genus has 9 species, 2 of them endemic. In this paper a synopsis of the genus in Mexico is presented; it includes a key to identify the species as well as distribution maps obtained from the specimens examined.
\end{abstract}

Key words: Asteraceae, Pluchea, Plucheeae, México.

\section{Introducción}

El género Pluchea Cass. pertenece a la tribu Plucheeae de la familia Asteraceae (Bremer 1994). Para algunos autores el género incluye alrededor de 40 especies (por ejemplo, Nesom 1989), aunque para otros, hasta 80 especies (por ejemplo, Bremer 1994). La mayoría se distribuye en el continente americano y otras las comparten África, Asia y Australia. Los miembros de este género, originalmente fueron ubicados por Cassini como parte de Conyza L. y en la tribu Vernonieae; posteriormente, fueron asignados a otros géneros de distintas tribus. Bentham (1873) fue el primero en ubicarlo como parte de su tribu Inuloideae, un grupo de géneros caracterizado por el receptáculo sin páleas, flores periféricas pistiladas y con corola filiforme, anteras caudadas en la base y las ramas del estilo con tricomas cortos en toda su extensión. Tradicionalmente se ha reconocido como un género perteneciente a la tribu Inuleae.

Anderberg (1989) propone la segregación de varios géneros de Inuleae en la nueva tribu Plucheeae, entre ellos Pluchea, el género tipo de la tribu; sus miembros se siguen caracterizando tal y como originalmente los definió Bentham. Anderberg (1991) analizó las relaciones filogenéticas entre los miembros de la tribu y ubica a Pluchea como el grupo hermano de Tessaria Ruiz et Pav., un género sudamericano. La tribu Plucheeae, como se define actualmente, está constituida por 28 géneros que incluyen alrededor de 220

Recibido: 15 marzo 2005; aceptado: 24 octubre 2005 especies (Bremer 1994).

El género ha sido estudiado taxonómicamente por varios autores. Robinson y Cuatrecasas (1973) evaluaron los límites genéricos entre Pluchea y Tessaria; Godfrey (1952) hizo la revisión de las especies norteamericanas de la sección Stylimnus, sección que incluye la mayoría de las especies mexicanas. Nesom (1989), además de adicionar especies nuevas del género a la flora de México, hizo una propuesta de clasificación infragenérica para las especies americanas. Por otra parte, Gillis (1977) y Khan y Jarvis (1989) discutieron diversos problemas nomenclaturales en el género y más recientemente Nesom (2004) discute otros aspectos nomenclaturales en el género y clarifica problemas de tipificación. En este trabajo se presenta una sinopsis del género Pluchea en México; el objetivo es presentar las especies existentes en el país, su nombre válido y los nombres con los cuales de una $\mathrm{u}$ otra manera han sido conocidas o citadas en la literatura y que en la actualidad se consideran sinónimos.

\section{Materiales y método}

Se estudió material herborizado que se encuentra depositado en diversos herbarios nacionales. Entre los principales se encuentran el Herbario Nacional del Instituto de Biología de la Universidad Nacional Autónoma de México (MEXU), el del Instituto de Botánica de la Universidad de Guadalajara (IBUG) y los herbarios del Instituto de Ecología 
en Pátzcuaro, Michoacán (IEB) y en Xalapa, Veracruz (XAL). Se revisó igualmente, aunque de manera parcial, la colección de la Universidad de Texas en Austin, Texas, Estados Unidos. A partir de la revisión crítica del material, se evaluaron diversos caracteres morfológicos que se consideraron de importancia taxonómica y de utilidad para la correcta identificación de las especies, elaborándose con ellos una clave de identificación. Las localidades de recolecta fueron georreferenciadas y con los datos de latitud y longitud se elaboraron mapas de distribución para cada especie.

\section{Resultados}

El género Pluchea registra 9 especies en México, que de acuerdo con la clasificación subgenérica de Nesom (1989) pertenecen a cuatro secciones (Cuadro 1). En México el género se distribuye ampliamente, pues se ha encontrado en 30 de los 32 estados en que se divide al país; solamente no ha sido registrado en los estados de Aguascalientes y Tlaxcala. Los estados de Oaxaca y Veracruz contienen el mayor número de especies (5), seguidos por Campeche, Quintana Roo y San Luis Potosí, cada uno con 4 especies. Los estados con 3 especies son Chiapas, Colima, Guerrero, Nayarit, Sinaloa, Sonora y Yucatán.

Cuadro 1. Secciones (Nesom 1989) donde se ubican las especies mexicanas del género Pluchea.

Pluchea Cass. sección Pluchea

1. Pluchea carolinensis (Jacq.) G. Don

2. Pluchea odorata (L.) Cass.

Pluchea Cass. sección Amplectifolium G.L. Nesom

3. Pluchea foetida (L.) DC.

4. Pluchea mexicana (Godfrey) G.L. Nesom

5. Pluchea rosea Godfrey

6. Pluchea yucatanensis G.L. Nesom

Pluchea Cass. sección Phalacroline A. Gray

\section{Pluchea sericea (Nutt.) Coville}

Pluchea Cass. sección Pterocaulis G.L. Nesom

8. Pluchea parvifolia (A. Gray) Godfrey

9. Pluchea salicifolia (Mill.) S.F. Blake

Pluchea carolinensis es la especie con más amplia distribución, registrada en 27 estados (Fig. 1). En segundo lugar se ubica $P$. salicifolia (21 estados, Fig. 4), seguida por P. odorata (16 estados, Fig. 3). Las especies con distribución restringida a un solo estado son P. mexicana, (San Luis Potosí, Fig. 2) y P. parvifolia (Baja California Sur, Fig. 2).

Pluchea Cass., Bull. Sci. Soc. Philom. Paris 31. 1817.

Hierbas perennes o arbustos, a veces aromáticos, por lo general pubescentes, a veces también con tricomas glandulares. Hojas simples, alternas, enteras, dentadas o serradas, pecioladas o sésiles y con las bases amplexicaules o decurrentes como alas. Cabezuelas heterógamas, dispuestas en cimas o corimbos, a veces secundariamente dispuestas en panículas; involucro campanulado a hemisférico, con brácteas imbricadas, graduadas, frecuentemente con tintes púrpura; receptáculo plano, desnudo; flores heterógamas, las periféricas pistiladas y fértiles, numerosas, en varias series, tubular-filiformes, con 3-5 lóbulos diminutos, usualmente provistos de glándulas y terminados en setas cortas; flores del disco hermafroditas pero funcionalmente masculinas, relativamente pocas, tubulares, con 5 lóbulos iguales, con tricomas cortos glandulares en la región de los lóbulos; anteras con las bases caudadas; ramas del estilo filiformes, frecuentemente sin separarse en las flores hermafroditas. Aquenios cilíndricos, fusiformes o clavados, 4-6-angulares, pubescentes o glabros; vilano formado por una serie de cerdas capilares.

Género de 40-80 especies, la mayoría americanas, con algunas especies en África, Asia y Australia; en México se reconocen 9.

Pluchea carolinensis (Jacq.) G. Don in Sweet, Hort. Brit., ed. 3: 350. 1839.

Conyza carolinensis Jacq., Collectanea 2: 271. 1789.

Arbustos hasta $3 \mathrm{~m}$ alto; tallos por lo general tomentosos, en ocasiones glabrescentes; hojas con pecíolos 1-3 cm largo, elípticas, ovadas u oblongas, 6-15 cm largo, 3-6 cm ancho, base cuneada, márgenes enteros a inconspicuamente dentados,

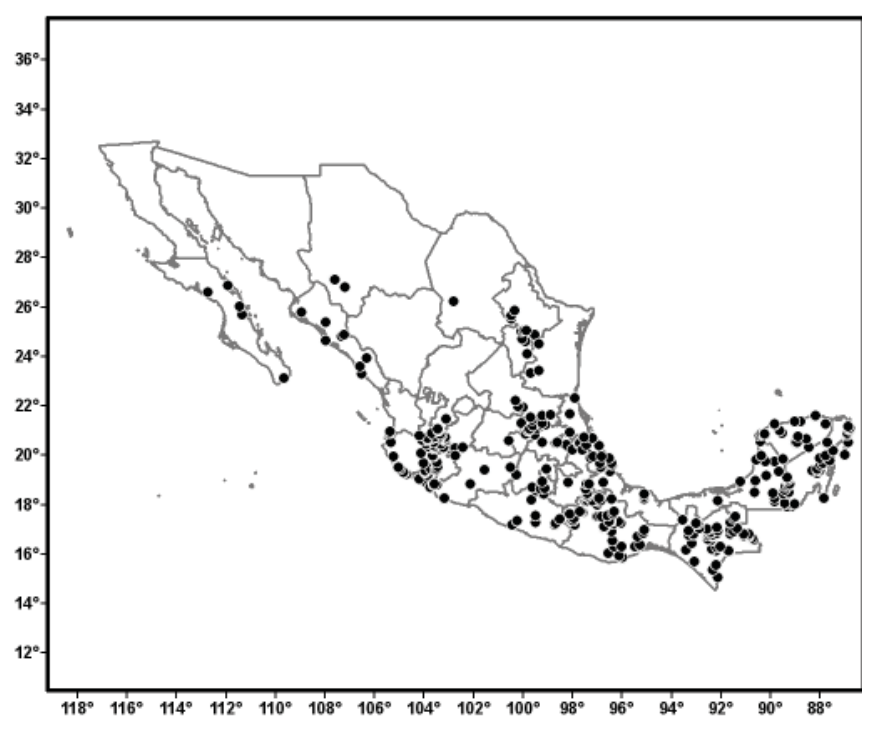

Figura 1. Distribución conocida de Pluchea carolinensis. 


\section{Clave para identificar las especies de Pluchea en México}

1. Arbustos; hojas grandes, hasta $15 \mathrm{~cm}$ largo, por lo general enteras o inconspicuamente dentadas, sedoso-pubescentes.

\section{Pluchea carolinensis}

1. Hierbas perennes o arbustos; hojas pequeñas, por lo general de menos de $6 \mathrm{~cm}$ largo, serradas o dentadas, rara vez dentadas y sedoso-pubescentes.

2. Tallos alados.

3. Cabezuelas de más de $8 \mathrm{~mm}$ largo y ancho; hojas lanceoladas a linear- lanceoladas, de tamaño más o menos uniforme hasta las inflorescencias; Baja California Sur.

3. Cabezuelas de menos de $8 \mathrm{~mm}$ largo y ancho; hojas lanceoladas, gradualmente Pluchea parvifolia haciéndose más pequeñas hacia las inflorescencias; no en la península de Baja California. Pluchea salicifolia

2. Tallos no alados.

4. Arbustos con hojas seríceas, 2-7 mm ancho, agudas o acuminadas en el ápice; cerdas del vilano de las flores hermafroditas dilatadas en el ápice; Baja California, Sonora.

Pluchea sericea

4. Hierbas perennes con hojas no seríceas, por lo general de más de $1 \mathrm{~cm}$ ancho, agudas u obtusas en el ápice; cerdas del vilano de las flores hermafroditas no dilatadas en el ápice.

5. Hojas conspicuamente pecioladas. Pluchea odorata

5. Hojas sésiles, por lo general amplexicaules o truncadas en la base.

6. Cabezuelas sésiles; hojas pubescentes, con tricomas tanto glandulares como no glandulares; San Luis Potosí.

Pluchea mexicana

6. Cabezuelas pedunculadas; hojas glabras o exclusivamente con tricomas no glandulares.

7. Pedúnculos solamente con tricomas estipitado glandulares; Campeche, Quintana Roo.

Pluchea yucatanensis

7. Pedúnculos con tricomas simples. no estipitado glandulares, a veces también con glándulas sésiles.

8. Flores rosas o púrpuras; pedúnculos y a veces también las brácteas involucrales densamente pubescentes; tricomas glandulares no evidentes; involucro 4-7 mm largo; Campeche, Quintana Roo, Veracruz, Yucatán.

\section{Pluchea rosea}

8. Flores blanco-cremosas; pedúnculos y brácteas involucrales no densamente pubescentes, con glándulas sésiles; involucro 7-9 mm largo; Oaxaca, Veracruz.

Pluchea foetida

ápice obtuso o ligeramente agudo, por lo general tomentosas en el envés; involucro campanulado a globoso, 5-6 mm largo; brácteas involucrales purpúreas, ovadas, ciliadas; flores rosadas o purpúreas.

Pluchea carolinensis ha tenido una historia nomenclatural azarosa. Godfrey (1952) llamó a esta especie $P$. odorata (L.) Cass.; sin embargo, Gillis (1977) discutió nuevamente su nomenclatura y llegó a la conclusión de que $P$. odorata era el nombre correcto del binomio tratado por Godfrey como P. purpurascens, indicando que el nombre correcto de este taxon era $P$. symphytifolia (Mill.) Gillis. Pero años más tarde Khan y Jarvis (1989) hacen una evaluación crítica de la literatura concerniente a esta especie y concluyen que el nombre de $P$. symphytifolia (Mill.) Gillis y su basiónimo
Conyza symphytifolia Mill., son nombres mal aplicados en el género Pluchea, y son más bien sinónimos nomenclaturales de Neurolaena lobata (L.) Cass., una especie de la tribu Heliantheae. Por todo esto, Khan y Jarvis concluyen que el nombre correcto de esta especie es $P$. carolinensis (Jacq.) G. Don y bajo este nombre debe ser conocido.

Hábitat: En zonas inundables, vegetación riparia o ruderal. Floración: registrada en floración todos los meses del año. Distribución fuera de México: Centroamérica, Sudamérica, Islas del Caribe.

Distribución en México (Fig.1): Baja California Sur: Carter 4897 (MEXU, TEX), Domínguez 512 (IEB); Campeche: Martínez 28955 (MEXU), Puch 230 (MEXU, UCAM), Schubert 1682 (MEXU), Ucán 4457 (MEXU), 
Zamora 6144 (UCAM); Chiapas: Cházaro 5323 (IBUG), Croat 47734 (MEXU), Espejo 2828 (HUMO), Luckow 2561 (MEXU, TEX), Lukasser 46 (MEXU), Webster 12912 (MEXU); Chihuahua: Bye 3604 (MEXU), Villarreal 17404 (IBUG); Coahuila: Carranza 1969 (MEXU, TEX); Colima: Cházaro 6443 (IEB, TEX), McVaugh 15686 (MEXU), Santana 1204 (IBUG), Villarreal 17603 (IBUG); Distrito Federal: Vibrans 7000 (MEXU); Guanajuato: Pérez 2893 (IBUG, MEXU), Rzedowski 52956 (IEB); Guerrero: Calónico 7019 (MEXU), Cruz 1979 (MEXU), Kruse 271 (MEXU), Soto 5097 (MEXU); Hidalgo: Hernández 5591 (MEXU); Jalisco: Calónico 7761 (MEXU), Cházaro 4380 (IBUG, MEXU), Pérez 1992 (MEXU), Villarreal 17817 (IBUG), Webster 15791 (MEXU); México: Matuda 28068 (MEXU); Michoacán: Daniel 4903 (MEXU, TEX), García 1738 (IEB), Soto 11148 (MEXU), Villarreal 14456 (IBUG); Morelos: Betancourt 140 bis (IBUG), Ortega 14 (MEXU), Vázquez 1753 (MEXU); Nayarit: Díaz 18642 (GUADA, IEB, TEX), González 821 (IBUG); Nuevo León: Hinton 21865 (IEB, TEX), Langman 2922 (MEXU), Lyonnet 3907 (MEXU), Sánchez 423 (MEXU); Oaxaca: Calzada 19699 (MEXU, TEX), Croat 65667 (MEXU), García 3103 (IEB, MEXU), Lorence 4823 (MEXU), Matuda 28496 (MEXU), Salinas F-3890 (MEXU); Puebla: Boege 1366 (MEXU), González 19 (IEB), Tenorio 21657 (MEXU), Villaseñor 273 (MEXU); Querétaro: Fernández 2769 (IBUG, MEXU), McVaugh 10372 (MEXU), Ortega 178 (IEB), Rzedowski 42629a, (IEB), Zamudio 2706 (MEXU); Quintana Roo: Cabrera 7802 (MEXU), Escalante 10 (MEXU, UCAM), Espejel 507 (XAL), Flores 8449 (MEXU), Sousa 11054 (MEXU), Ucán 375 (MEXU); San Luis Potosí: Alcorn 2502 (MEXU, TEX), Gómez 948 (IBUG, MEXU), Medellín 1155 (MEXU), Rzedowski 8668 (MEXU); Sinaloa: Breckon 507 (MEXU), Gentry 5931 (MEXU), Seigler 11667 (MEXU), Tenorio 8454 (MEXU, TEX); Tabasco: Calzada 2220 (MEXU, UCAM), Guadarrama 5100 (MEXU), Zamudio 748 (MEXU); Tamaulipas: Dorr 2365 (MEXU, TEX), Mora 7843 (MEXU); Veracruz: Conzatti 6004 (MEXU), Durán 209 (IEB, MEXU, XAL), Jarquin 20 (MEXU, XAL), Ventura 15027 (MEXU, XAL); Yucatán: Carnevali 5375 (MEXU, UCAM), Chan 2200 (MEXU), Durán 1744 (MEXU), Simá 59 (MEXU, XAL); Zacatecas: Estrada s. n. (IBUG).

Pluchea foetida (L.) DC., Prodr. 5: 452.1836

Baccharis foetida L., Sp. Pl. ed. 1: 861. 1753

Conyza amplexicaulis Michx., Fl. Bor. Amer. 2: 126. 1803, no Lam., 1786.

Pluchea bifrons DC., Prodr. 5: 451. 1836

Pluchea tenuifolia Small, Man. Souheast. Fl. 1399. 1933. Hierbas perennes $50-90 \mathrm{~cm}$ alto; tallos glabros a pubescentes, los tricomas no glandulares; hojas sésiles, oblanceoladas, 3$10 \mathrm{~cm}$ largo, $1.0-1.5 \mathrm{~cm}$ ancho, base cuneada o truncada, semiamplexicaule, márgenes serrados, ápice agudo; involucro campanulado, 7-9 mm largo; brácteas involucrales verdosas, lanceolado-ovadas, inconspicuamente pubescentes; flores blanco cremoso.

Hábitat: Principalmente en zonas inundadas, claros de bosques tropicales y dunas costeras.

Floración: registrada en floración en el mes de mayo.

Distribución fuera de México: Estados Unidos, República Dominicana.

Distribución en México (figura 2): Oaxaca: López 122 (MEXU). Veracruz: Calzada 5964 (TEX).

Pluchea mexicana (Godfrey) G.L. Nesom, Phytologia 67: 159. 1989

Pluchea rosea Godfrey var. mexicana Godfrey, J. Elisha Mitchell Sci. Soc. 68: 269. 1952.

Hierbas perennes $40-70 \mathrm{~cm}$ alto; tallos pubescentes, con tricomas multicelulares no glandulares y tricomas glándulares, tanto sésiles como estipitados; hojas sésiles, lanceoladas, oblanceoladas u oblongas, 2.5-5.0 cm largo, 7$20 \mathrm{~mm}$ ancho, base amplexicaule, márgenes serrados, ápice agudo; involucro campanulado, 8-9 $\mathrm{mm}$ largo; brácteas involucrales rosadas o púrpuras, lanceoladas, pubescentes, con tricomas glandulares; flores rosadas o púrpura.

Hábitat: vegetación halófila.

Floración: registrada en floración de junio a agosto.

Distribución fuera de México: Especie endémica de México.

Distribución en México (Fig. 2): San Luis Potosí: Palmer 75 (CM, F, MO, US), Purpus s. n. (F, US), Pringle 3813 (F, GH, MEXU, MU, NY, PH, US).

Pluchea odorata (L.) Cass., Dict. Sci. Nat. 42: 3. 1826.

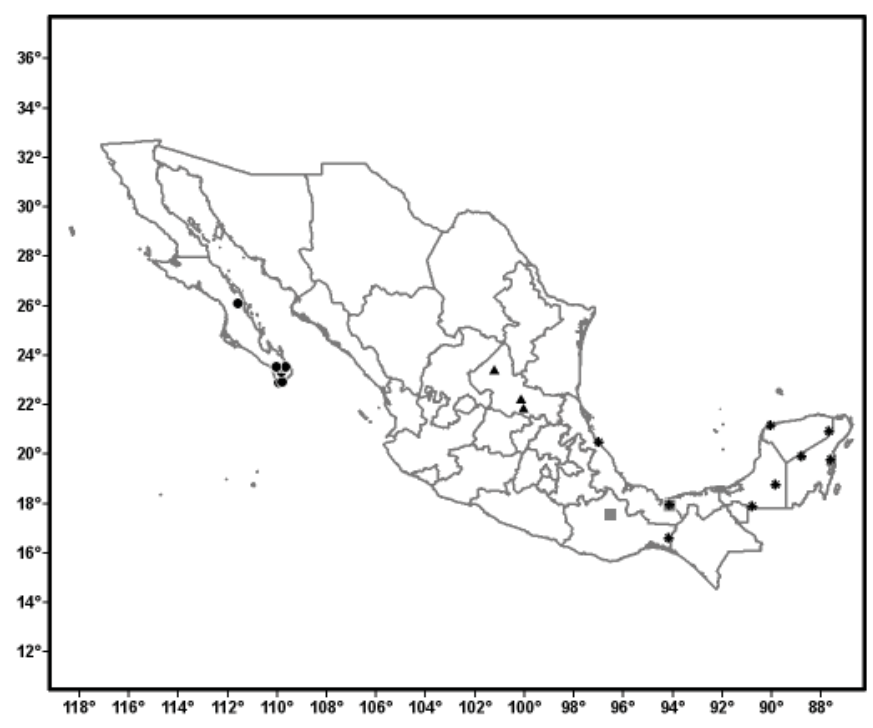

Figura 2. Distribución conocida de Pluchea foetida (cuadros), $P$. mexicana (triángulos), $P$. parvifolia (círculos) y $P$. rosea (asteriscos). 
Conyza odorata L., Syst. Nat. ed. 10, 1213. 1759.

Conyza purpurascens Sw., Prodr. 112. 1788.

Pluchea purpurascens (Sw.) DC., Prodr. 5: 452. 1836.

Hierbas perennes $40-90 \mathrm{~cm}$ alto; tallos glabros o a veces pubescentes en la porción superior, los tricomas no glandulares; hojas con pecíolos 1.0-2.5 cm largo, ovadas, elípticas o lanceoladas, $2.5-6.0 \mathrm{~cm}$ largo, 1.2-3.0 cm ancho, base atenuada, márgenes serrados o dentados, ápice agudo; involucro campanulado, 5-7 mm largo; brácteas involucrales púrpuras o rosadas, oblongas, puberulentas; flores rosadas o púrpura.

Hábitat: zonas inundables en bosque tropical caducifolio, bosque tropical perennifolio, bosque tropical subcaducifolio, dunas costeras, manglar, matorral xerófilo, vegetación riparia.

Floración: registrada en floración todos los meses del año. DistribuciónfueradeMéxico:Estados Unidos,Centroamérica, Sudamérica e Islas del Caribe.

Distribución en México (Fig. 3): Campeche: Cabrera 13995 (MEXU), Chan 4915 (MEXU), Flores 9764 (MEXU), Góngora 543 (MEXU), Martínez 27964 (MEXU), Matuda 3859 (MEXU), Ocaña 216 (MEXU), Zamora 5114 (MEXU, UCAM); Chiapas: Matuda 16278 (MEXU); Coahuila: Carranza 3476 (IEB), Villarreal 3198 (ANSM, MEXU); Colima: Díaz 3305 (GUADA), Gregory 314 (MEXU); Guerrero: Boege 822 (MEXU), Castillo 6569 (MEXU), Fonseca 146 (MEXU), Freeland 213 (MEXU), Ojeda 73 (MEXU); Nayarit: González 5538 (MEXU), King 3697 (MEXU, TEX); Nuevo León: Bye 28333 (MEXU), Carranza 2638 (MEXU), Sánchez 420 (MEXU); Oaxaca: Castillo 9749 (MEXU), Elorsa 4255 (MEXU), Illescas 4 (MEXU); Quintana Roo: Boege 3255 (MEXU), Cabrera

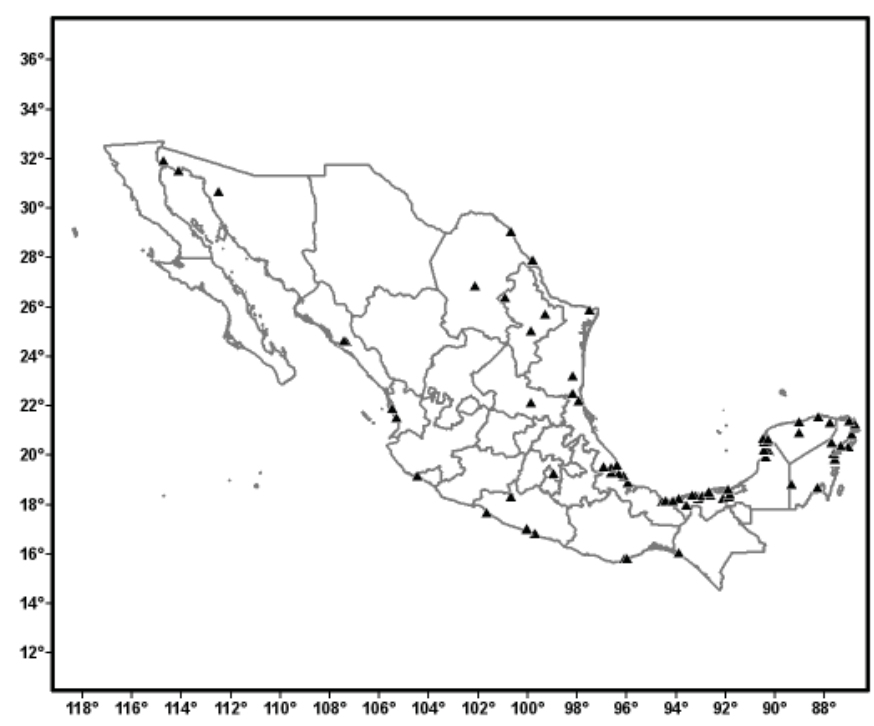

Figura 3. Distribución conocida de Pluchea odorata.
4754 (MEXU), Chan 2710 (MEXU), Davidse 20631 (MEXU), Durán 704 (MEXU), Moreno 816 (MEXU), Olmsted 42 (MEXU), Puch 23 (MEXU, UCAM), Ucán 1061 (MEXU), Villanueva 618 (MEXU); San Luis Potosí: Takaki 292 (MEXU); Sinaloa: Keil 15054 (MEXU, TEX), Vega 1226 (MEXU); Sonora: Ezcurra s. n. (MEXU), Felger 85-1048 (MEXU, TEX); Tabasco: Barlow 28/7D (MEXU), Cowan 3104 (MEXU, TEX), Curiel 92 (IBUG), González 4 (MEXU), Guadarrama 6445 (MEXU), Novelo 4157 (MEXU), Ventura 20471 (IBUG, MEXU), Zamudio 268 (IBUG, MEXU); Tamaulipas: Dressler 2106 (MEXU), González 13023 (MEXU), Mora 794 (MEXU), Pringle s. $n$. (MEXU); Veracruz: Gutiérrez 1882 (IEB, XAL), King 1139 (MEXU, TEX), Orcutt 3160 (MEXU, TEX, XAL), Sinaca 2081 (MEXU), Vargas 520 (IEB, XAL), Vázquez 1338 (IBUG), Ventura 8366 (IEB, MEXU, XAL), Villaseñor 965 (MEXU, XAL), Zamora 463 (IEB, MEXU, XAL); Yucatán: Cabrera 13744 (MEXU), Chan 6992 (MEXU), Durán 1519 (MEXU), Gaumer 1002 (MEXU), Simá 1403 (MEXU).

Pluchea parvifolia (A. Gray) Godfrey, J. Elisha Mitchell Sci. Soc. 68: 252. 1952.

Pluchea subdecurrens Cass. var. parvifolia A. Gray, Proc. Amer. Acad. Arts 5: 160. 1861.

Pluchea adnata (Humb. et Bonpl.) C. Mohr var. parvifolia (A. Gray) S.F. Blake, Contr. U. S. Nat. Herb. 23: 1510. 1926.

Pluchea salicifolia (Mill.) S.F. Blake var. parvifolia (A. Gray) S.F. Blake, J. Wash. Acad. Sci. 21: 328. 1931.

Hierbas perennes $80-150 \mathrm{~cm}$ alto; tallos pubescentes y con tricomas glandulares; hojas sésiles, lanceoladas o linearlanceoladas, 5-8 cm largo, 6-16 cm ancho, base decurente como ala sobre el tallo, márgenes serrados o dentados, ápice agudo; involucro turbinado, 8-12 $\mathrm{mm}$ largo; brácteas involucrales púrpuras, lanceoladas, pubescentes, los tricomas glandulares; flores blanco cremoso o rosadas.

Hábitat: principalmente en vegetación riparia dentro del bosque de encino y del bosque tropical caducifolio.

Floración: registrada en floración de enero a agosto.

Distribución fuera de México: Especie endémica de México.

Distribución en México (Fig. 2): Baja California Sur: Carter 3308 (MEXU), Domínguez 222 (MEXU), Gentry 4343 (MEXU), Gilmartin 1859 (MEXU), León 2501 (MEXU), Thomas 7814 (MEXU), Webster 19529 (MEXU, TEX), Xantus 53 (K fotografía en MEXU, NY fotografía en MEXU)

Pluchea rosea Godfrey, J. Elisha Mitchell Sci. Soc. 68: 266. 1952.

Hierbas perennes $40-80 \mathrm{~cm}$ alto; tallos glabros a pubescentes, los tricomas simples, con glándulas sésiles y glóbulos de exudado resinoso; hojas sésiles, oblanceoladas a oblongas, 2.5-7.0 $\mathrm{cm}$ largo, 5-30 $\mathrm{mm}$ ancho, base amplexicaule, 
márgenes serrados, ápice agudo o ligeramente obtuso; involucro campanulado, turbinado-campanulado o turbinado 4-7 mm largo; brácteas involucrales rosadas o púrpuras, lanceolado-ovadas, pubescentes, los tricomas glandulares inconspicuos; flores rosadas o púrpura.

Hábitat: zonas inundables de bosque de pino-encino, bosque tropical subcaducifolio, dunas costeras, sabana.

Floración: registrada en floración de enero a octubre.

Distribución fuera de México: Estados Unidos, islas del Caribe.

Distribución en México (Fig. 2): Campeche: Chan 3754 (MEXU), Puch 1327 (MEXU); Oaxaca: Maya 2686 (MEXU, TEX); Quintana Roo: Chan 786 (MEXU), Durán 254 (MEXU), Rico 392 (MEXU), Steere 2407 (F, US); Veracruz: Orozco 161 (MEXU, XAL); Yucatán: Estrada 333 (MEXU).

Pluchea salicifolia (Mill.) S.F. Blake, Contr. U. S. Nat. Herb. 26: 237. 1930

Conyza salicifolia Mill., Gard. Dict. ed. 8, Conyza No. 6. 1768 .

Baccharis adnata Humb. et Bonpl. ex Willd., Enum. Plant. Hort. Berol. 870. 1809.

Conyza adnata (Humb. et Bonpl.) Kunth, in H.B.K. Nov. Gen. et Sp. 4: 74. 1820.

Pluchea subdecurrens Cass., Dict. Sci. Nat. 42: 4. 1826.

Pluchea subdecurrens Cass. var. canescens A. Gray, Proc. Amer. Acad. Arts 5: 82. 1861.

Pluchea adnata (Humb. et Bonpl.) Mohr, Contr. U. S. Nat. Herb. 6: 790. 1901.

Pluchea adnata (Humb. et Bonpl.) Mohr var. canescens (A. Gray) S.F. Blake, Contr. U. S. Nat. Herb. 23: 1510. 1926.

Pluchea salicifolia (Mill.) S.F. Blake var. canescens (A. Gray) S.F. Blake, J. Wash. Acad. Sci. 21: 328. 1931.

Hierbas perennes 1-2(-3) $\mathrm{m}$ alto; tallos densamente pubescentes, por lo general canescentes y con tricomas glandulares; hojas sésiles, lanceoladas o lineares, 6-10 cm largo, 4-20 $\mathrm{mm}$ ancho, base decurente como ala sobre el tallo, márgenes serrados o dentados, rara vez enteros, ápice agudo; involucro campanulado, 4-6 mm largo; brácteas involucrales púrpuras, lanceoladas, pubescentes y con tricomas glandulares; flores rosadas o blanco-cremoso.

Hábitat: en muchas comunidades vegetales, incluyendo zonas inundables y vegetación acuática; en muchas de ellas como ruderales.

Floración: registrada en floración todos los meses del año.

Distribución fuera de México: Guatemala.

Distribución en México (Fig. 4): Chiapas: Croat 46288 (MEXU), Miranda 5897 (MEXU); Chihuahua: Lebgue 2088 (MEXU); Colima: Carvajal 369 (IBUG), Díaz 9955 (GUADA), Lorence 3799 (MEXU), Rothschild 111 (HUMO, MEXU), Sanders 10684 (MEXU); Durango:
Breedlove 24541 (CIIDIR, MEXU); Guanajuato: Kishler 591 (MEXU), Ventura 7397 (IBUG, MEXU); Guerrero: Calónico 13894 (MEXU), Catalán 655 (MEXU), Diego 2498 (MEXU), Moreno 777 (MEXU), Prather 1192 (MEXU); Hidalgo: Argüelles 3054 (MEXU), Hernández 5675 (MEXU); Jalisco: Carrillo 3903 (GUADA), Flores 1785 (MEXU, XAL), Machuca 7103 (IBUG, TEX , XAL), Rodríguez 1204 (HUMO, IBUG), Sanders 10340 (MEXU, TEX), Villarreal 13673 (IBUG); México: Hinton 3025 (MEXU), Matuda 27432 (MEXU); Michoacán: Castro 456 (HUMO, MEXU), Díaz 6157 (IEB, MEXU, XAL), Lyonnet 382 (MEXU), Matuda 26008 (MEXU), Rzedowski 41218 (IEB, MEXU), Soto 6224 (MEXU), Steinmann 4206 (MEXU); Morelos: Pringle 8003 (MEXU, TEX), Vázquez 2103(MEXU); Nayarit: Téllez 11873 (MEXU); NuevoLeón: Hinton 22085 (TEX , XAL), Palmer 546 (K fotografía en MEXU), Pringle 10198 (MEXU); Oaxaca: Calzada 22452 (MEXU), Conzatti 2490 (MEXU), Flores 1270 (MEXU), García 3668 (MEXU), Guízar 4405 (MEXU), Maya 4244 (MEXU, TEX), Villaseñor 910 (MEXU, TEX); Puebla: Hardison 101 (MEXU, TEX), Miranda 2505 (MEXU), Mota 162 (MEXU), Tenorio 5106 (MEXU); Querétaro: Carranza 1613 (IBUG, MEXU), Fernández 2857 (MEXU), Hernández 9278 (MEXU), López 537 (MEXU), Rzedowski 43383 (IBUG, IEB), Zamudio 2019 (IEB); San Luis Potosí: Chemin 122 (MEXU), Sánchez s. n. (MEXU); Sinaloa: Jasso 5 (MEXU), Vega 3221 (MEXU), White 5052 (MEXU, TEX); Sonora: Búrquez 96-137 (MEXU), Felger 84-144 (MEXU), Reina 97-465 (MEXU, TEX), Steinmann 93-126 (MEXU), Varela 97-100 (MEXU, TEX) ; Tamaulipas: González 14513 (MEXU), Mora 5722 (MEXU); Veracruz: Castillo 3099 (IEB, XAL), Dorantes 527 (MEXU, XAL), Lot 309 (MEXU, XAL), Nee 26110 (MEXU, TEX , XAL),

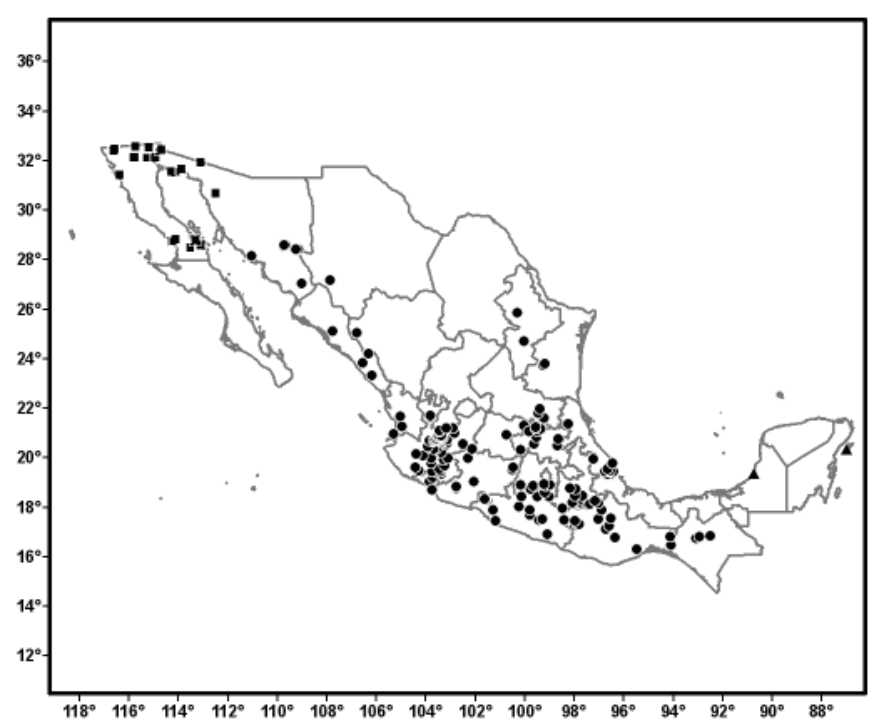

Figura 4. Distribución conocida de Pluchea salicifolia (círculos), $P$. sericea (cuadros) y P. yucatanensis (triángulos). 
Ventura 12439 (IEB, MEXU, XAL); Zacatecas: Flores s. $n$. (IBUG), Enríquez 7275 (HUAZ).

Pluchea sericea (Nutt.) Coville, Contr. U. S. Nat. Herb. 4: 128. 1893.

Polypappus sericeus Nutt., J. Acad. Nat. Sci. Philad. ser. 2, 1: 178. 1847.

Tessaria borealis Torr. et A. Gray ex A. Gray, Pl. Fendl. 75. 1849.

Berthelotia sericea (Nutt.) Rydb., Bull. Torrey Bot. Club. 33: 154. 1906.

Tessaria sericea (Nutt.) Shinners, Sida 3: 122. 1967. Arbustos 50-120 cm alto, aromáticos; tallos densamente pubescentes, los tricomas no glandulares, seríceos; hojas sésiles, seríceas, lineares a lanceoladas, 1-6 cm largo, 2-7 $\mathrm{mm}$ ancho, base truncada, márgenes enteros, ápice agudo o acuminado; involucro campanulado, 4-5 mm largo; brácteas involucrales, oblongas, seríceas o tomentosas; flores púrpura.

Hábitat: dunas costeras, matorral xerófilo, vegetación halófila,vegetación riparia, en suelos inundables o como parte de la vegetación acuática.

Floración: registrada en floración de marzo a diciembre.

Distribución fuera de México: Estados Unidos.

Distribución en México (Fig. 4): Baja California Norte: Boyd 5668 (MEXU), Charlton 1375 (MEXU), Felger 77-5 (MEXU), Tenorio 13449 (MEXU), Thorne 62113 (MEXU), Wiggins 15735 (MEXU, TEX); Sonora: Ezcurra s. $n$. (MEXU), Felger 85-1045 (MEXU, TEX), Reina 97-534 (MEXU, TEX), Webster 24242 (MEXU).

Pluchea yucatanensis G. L. Nesom, Phytologia 67: 159. 1989.

Hierbas perennes $20-60 \mathrm{~cm}$ alto; tallos glabros pero con glándulas sésiles; hojas algo coriáceas y suculentas, sésiles, oblongo-ovadas, 3-5 cm largo, 6-19 mm ancho, base amplexicaule, márgenes serrulados, ápice agudo; involucro turbinado a campanulado, 4-5 mm largo; brácteas involucrales púrpuras, lanceoladas, solamente con tricomas glandulares; flores rosadas o blanco cremoso.

Hábitat: sabana, vegetación acuática.

Floración: registrada en floración en el mes de julio.

Distribución fuera de México: Estados Unidos (según Nesom (1989) adventicia).
Distribución en México (Fig. 4): Campeche: Steere 1844

(LL-TEX, US); Quintana Roo: Lot 848 (MEXU).

\section{Agradecimientos}

Agradecemos a Thomas L. Wendt su ayuda para resolver algunas dudas tanto bibliográficas como de material depositado en el herbario de la Universidad de Texas en Austin (TEX) y a Verónica Juárez y Rosalinda Medina la lectura del manuscrito y sus comentarios. La Comisión Nacional para el Conocimiento y Uso de la Bodiversidad (CONABIO) apoyó con recursos económicos (proyecto BE007), Enrique Ortiz elaboró los mapas de distribución de las especies y Guadalupe Segura ayudó en la consulta, manejo y revisión del material en el Herbario Nacional (MEXU).

\section{Literatura citada}

Anderberg, A. A. 1989. Phylogeny and reclassification of the tribe Inuleae (Asteraceae). Canadian Journal of Botany 67: 2277-2296.

Anderberg, A. A.. 1991. Taxonomy and phylogeny of the tribe Plucheeae (Asteraceae). Plant Systematics and Evolution 176: 145-177.

Bentham, G. 1873. Compositae. In Genera Plantarum, vol. 2. G. Bentham y J. D. Hooker (eds.). Lovell Reeve, Londres. p. 163-533.

Bremer, K. 1994. Asteraceae. Cladistics and classification. Timber Press. Portland, Oregon. 752 p.

Gillis, W. T. 1977. Pluchea revisited. Taxon 26: 587-591.

Godfrey, R. K. 1952. Pluchea, section Stylimnus, in North America. Journal of the Elisha Mitchell Science Society 68: 238-279.

Kahn, R. y C. E. Jarvis. 1989. The correct name for the plant known as Pluchea symphytifolia (Miller) Gillis (Asteraceae). Taxon 38: 659-662.

Nesom, G. L. 1989. New species, new sections, and a taxonomic overview of American Pluchea (Compositae: Inuleae). Phytologia 67: 158-167.

Nesom, G. L. 2004. Notes on typification in Pluchea (Asteraceae: Plucheae). Sida 21: 59-64.

Robinson, H. y J. Cuatrecasas. 1973. The generic limits of Pluchea and Tessaria. Phytologia 27: 277-285. 